\title{
VARIACIÓN INTRAESPECÍFICA EN LA PROBÓSCIDE DE POLYMORPHUS TROCHUS (POLYMORPHIDA: POLYMORPHIDAE) DE DOS ESPECIES DE AVES DULCEACUÍCOLAS (GRUIFORMES: RALLIDAE) EN EL ESTADO DE MÉXICO
}

\author{
INTRAESPECIFIC VARIATION IN THE PROBOSCIS OF POLYMORPHUS TROCHUS \\ (POLYMORPHIDA: POLYMORPHIDAE) IN TWO SPECIES OF FRESHWATER BIRDS \\ (GRUIFORMES: RALLIDAE) IN THE STATE OF MEXICO
}

\author{
Carmen CABAllero-Viñas ${ }^{1}$, Petra SÁnCheZ-NAVA ${ }^{1}$, Carlos AgUilar-ORTIGOZA ${ }^{2}$, Felipe \\ RODRÍGUEZ-ROMERO ${ }^{*}$
}

\begin{abstract}
${ }^{1}$ Laboratorio de Sistemas Biosustentables, Facultad de Ciencias, Universidad Autónoma del Estado de México. Campus Universitario "El Cerrillo" El Cerrillo Piedras Blancas, Carretera Toluca-Ixtlahuaca Km 15.5; CP 50200, Toluca, Estado de México, México. <c-caballero@live.com.mx>; 〈psn@uaemex.mx>; 〈fjrr@uaemex.mx>

${ }^{2}$ Facultad de Ciencias, Universidad Autónoma del Estado de México. Campus Universitario "El Cerrillo", El Cerrillo Piedras Blancas, Carretera Toluca-Ixtlahuaca Km 15.5; CP 50200, Toluca, Estado de México, México. 〈aguilarcj@uaemex.mx> *Autor de correspondencia: <fjrr@uaemex.mx>
\end{abstract}

Recibido: 03/07/2018; aceptado: 19/09/2019; publicado en línea: 30/09/2019

Editor responsable: Ricardo Rodríguez Estrella

Caballero-Viñas, C., Sánchez-Nava, P., Aguilar-Ortigoza, C., Rodríguez-Romero, F. (2019) Variación intraespecífica en la probóscide de Polymorphus trochus (Polymorphida: Polymorphidae) de dos especies de aves dulceacuícolas (Gruiformes: Rallidae) en el Estado de México. Acta Zoológica Mexicana (nueva serie), 35, 1-12. https://doi.org/10.21829/azm.2019.3502057

RESUMEN. Se utilizaron análisis de morfometría geométrica y lineal para evaluar la variación morfológica de la probóscide de Polymorphus trochus de Fulica americana y Gallinula galeata, ambas aves residentes del Humedal Chimaliapan, Estado de México. Los análisis de morfometría geométrica revelaron la existencia de distintos morfotipos de acuerdo con la especie de hospedero, el microhábitat, el sexo y el estado de desarrollo del parásito. Las hembras adultas se ubicaron en el íleon y presentaron una probóscide ensanchada, a diferencia de las hembras jóvenes, que se encontraron en el yeyuno y tuvieron una probóscide estrecha, muy similar a los machos; esto sugiere diseños distintos que se ajustan al movimiento migratorio de este grupo. La morfometría lineal también indicó diferencias en la longitud de la probóscide y los ganchos; además se identificó que las hembras presentan mayor tamaño de estas estructuras, como una adaptación hacia una fijación permanente para una adecuada maduración y posterior liberación de los huevos. De acuerdo con el presente estudio, la reproducción y la estructura del microhábitat son dos características importantes que influyen en el tamaño y forma de la probóscide. Adicionalmente, se indica que existen diferencias estadísticas en el dimorfismo sexual en diferentes niveles: forma y tamaño de la probóscide y longitud de los ganchos, datos no reportados para esta especie con anterioridad.

Palabras clave: Polymorphus trochus; Acanthocephala; probóscide; dimorfismo sexual; morfometría geométrica 
Caballero-Viñas et al.: Variación intraespecífica en la probóscide en

Polymorphus trochus

Caballero-Viñas, C., Sánchez-Nava, P., Aguilar-Ortigoza, C., Rodríguez-Romero, F. (2019) Intraespecific variation in the proboscis of Polymorphus trochus (Polymorphida: Polymorphidae) in two species of freshwater birds (Gruiformes: Rallidae) in the State of Mexico. Acta Zoológica Mexicana (nueva serie), 35, 1-12. https://doi.org/10.21829/azm.2019.3502057

\begin{abstract}
Geometric and linear morphometric analyses were used to evaluate the morphological variation of the proboscis of Polymorphus trochus of Fulica americana and Gallinula galeata, both resident birds of Chimaliapan Wetland, State of Mexico. The geometric morphometric analysis revealed the existence of different morphotypes according to host species, microhabitat, sex and state of development of the parasite. The adult females were located in the ileum and showed an enlarged proboscis, unlike the young females, which were found in the jejunum and had a narrow proboscis, very similar to the males; this suggests different designs that fit the migratory movement of this group. Linear morphometric also indicated differences in the length of the proboscis and the hooks; it was also identified that the females present a larger size of these structures, as an adaptation towards a permanent attachment to an adequate maturation and subsequent release of the eggs. According to the present study, the reproduction and structure of the microhabitat are two important traits that influence the size and shape of the proboscis. Additionally, it is indicated that there are statistical differences in the sexual dimorphism in different levels: shape and size of the proboscis and length of the hooks, data not reported for this species before.
\end{abstract}

Key words: Polymorphus trochus; Acanthocephala; proboscis; sexual dimorphism; geometric morphometric

\title{
INTRODUCCIÓN
}

Todo organismo posee una morfología de modo que satisfaga sus múltiples demandas funcionales (Aznar et al., 2002); un ejemplo de ello son los parásitos, que tienden a optimizar sus características de historia de vida para adaptarse localmente al entorno proporcionado por sus hospederos. Para lograr este objetivo, los organismos moldean sus características morfológicas, fisiológicas, conductuales, entre otras (Bush, 2009). En un contexto evolutivo y funcional, diversos aspectos de la morfología del Phyllum Acanthocephala (gusanos de cabeza espinosa), son modificados por los requerimientos de una fijación adecuada al microambiente (Petrochenko, 1956), por lo que poseen estructuras de anclaje que varían significativamente entre especies (Poulin, 2007). Otros autores han documentado diferencias entre especies en cuanto al tamaño, número, distribución y patrón de crecimiento en estructuras de fijación primaria (e.g. ganchos y probóscide) y secundaria (e.g. espinas troncales), así como en el cuerpo del gusano (Poulin, 2007; Hernández-Orts et al., 2012); también existen estudios que demuestran la existencia de variación fenotípica en estas estructuras a nivel intraespecífico (Aznar et al., 1999; Aznar et al., 2002) e intrasexual (HernándezOrts et al., 2012); razones por las cuales se argumenta que los cambios morfológicos aparentemente simples, pueden haber desencadenado una cascada de modificaciones, así como nuevas funciones durante la adaptación de los acantocéfalos (Aznar et al., 1999).

No obstante lo anterior, estudios previos se han basado únicamente en morfometría lineal, que hace imposible dividir el tamaño y la forma de estructuras anatómicas para análisis separados, además de no proporcionar vías gráficas para localizar y comparar esta variación; entregándonos herramientas limitadas debido a que el tamaño, como dato numérico, es la base de sus análisis y no considera la geometría del objeto como una representación abstracta de la estructura (Corti et al., 2001; Toro et al., 2010). Estos límites han sido superados por la introducción de la morfometría geométrica, que tiene su origen en la estadística multivariada de datos geométricos (Rohlf \& Marcus, 1993; Corti, 2001; Toro et al., 2010) y en donde la forma del objeto en estudio no se describe en términos de sus dimensiones, sino de la relación espacial entre sus partes y por lo tanto, la información relativa a la geometría de los objetos se puede recuperar; esto 
permite la visualización de los cambios morfológicos y señalar a posteriori, tanto estadística como visualmente, qué región o estructura está siendo modificada (Toro et al., 2010).

En el presente estudio, se utilizaron los dos tipos de morfometría para examinar la variación intraespecífica en Polymorphus trochus de dos aves acuáticas: Gallinula galeata y Fulica americana, ambas residentes del humedal Chimaliapan, Estado de México. Los análisis se enfocaron exclusivamente en los morfotipos y medidas de la probóscide, así como longitud de los ganchos, ya que estos caracteres son considerados las estructuras de fijación primaria por ser las principales responsables de mantener al parásito dentro del lumen intestinal. Se describe la anatomía del sistema de fijación de Polymorphus trochus, que es el acantocéfalo más común de la gallareta americana y raramente se encuentra en patos de Norteamérica (McDonald, 1988). Estos gusanos presentan un cuerpo ligeramente ensanchado en la parte anterior y cubierto con espinas cuticulares, el cuello es semi-cilíndrico, con forma de barril, sin espinas. Las hembras son de mayor longitud que los machos; además, esta especie tiene la particularidad de que su probóscide muestra dimorfismo sexual: es piriforme, muy hinchada en la base y con la porción terminal que se estrecha en una punta lisa en hembras y cilindroide pero con un ligero ensanchamiento terminal en machos (Van Cleave, 1945; Petrochenko, 1958; Amin, 1992).

\section{MATERIALES Y MÉTODOS}

Sitio de estudio: colecta de hospederos y parásitos. Las aves acuáticas se obtuvieron por donación de cazadores locales durante el periodo comprendido entre febrero de 2016 y abril de 2017, en el Humedal Chimaliapan (19 $11^{\prime} 20^{\prime \prime}$ N; 99 29'30' O; Martínez-Haro et al., 2012) perteneciente al Ejido de San Pedro Tultepec, en el municipio de Lerma, Estado de México (Fig. 1). Se utilizaron las guías de campo de Preston (1998) y de Van Perlo (2006) para la identificación taxonómica de las aves.

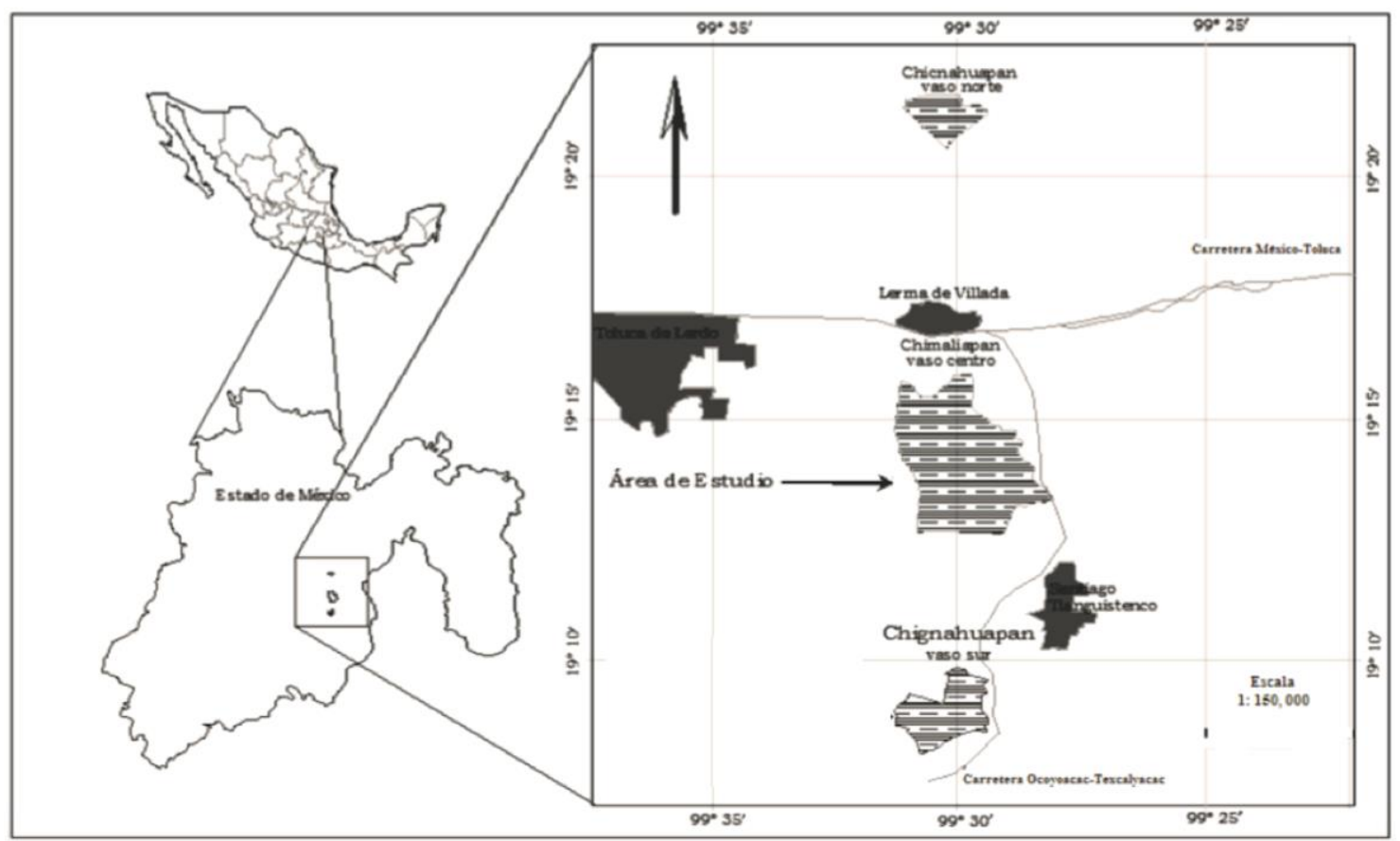

Figura 1. Ubicación del Humedal Chimaliapan (flecha pequeña). Tomado y modificado de Colón-Quezada (2009).

Las aves se trasladaron inmediatamente al laboratorio de Sistemas Biosustentables de la UAEMex, en donde se realizaron las necropsias y los exámenes helmintológicos de los intestinos de cada ave mediante 
Caballero-Viñas et al.: Variación intraespecífica en la probóscide en

Polymorphus trochus

el uso de un microscopio estereoscópico Olympus SZX7. Los acantocéfalos se extrajeron con ayuda de agujas de disección con cuidado de preservar la probóscide; a continuación, se colocaron en agua destilada y fueron sometidos a refrigeración por 24 horas para permitir la eversión de la probóscide (SalgadoMaldonado, 2009). Se fijaron con formol caliente al $4 \%$ y se conservaron en alcohol al $70 \%$ para posteriormente teñirlos con Paracarmín de Meyer y montarlos en preparaciones permanentes con bálsamo de Canadá (Salgado-Maldonado, 2009).

La identificación de especies parásitas se realizó de manera tradicional mediante las claves de Yamaguti (1963), McDonald (1988) y Amin (1992). El sexo de cada individuo se distinguió con base en el dimorfismo que presenta la probóscide de esta especie, así como a la observación de los órganos sexuales (Van Cleave, 1945; Petrochenko, 1958; Amin, 1992).

Se utilizaron un total de $81 P$. trochus: 65 de $F$. americana en dos diferentes microhábitats, que corresponden a dos diferentes zonas del intestino delgado (1: yeyuno y 2: íleon); y 16 de G. galeata, ubicados únicamente en íleon (microhábitat 2). Las zonas del intestino se reconocieron mediante análisis microscópicos y macroscópicos (Sisson \& Grossman, 2002).

Del total de gusanos, se eligieron 51 para los análisis de morfometría geométrica y 34 para los análisis de morfometría lineal; para ambos se utilizaron fotografías de la probóscide de los acantocéfalos tomadas a objetivo de 10x con un microscopio óptico Olympus BX41 con cámara integrada Olympus E620. Ambos análisis se centraron específicamente en comparar los patrones de forma y variación del tamaño en relación con el sexo del parásito, la especie de hospedero y el microhábitat de los parásitos.

Morfometría lineal. Se utilizó el programa ImageJ para obtener las medidas lineales de largo y ancho de la probóscide de cada individuo, así como de la longitud y base de cada gancho de una hilera lateral completa (Petrochenko, 1956). La dimensionalidad de las variables obtenidas se redujo mediante el Análisis de Componentes Principales; posteriormente, una prueba de ANOVA permitió comparar las variables por hospedero, microhábitat y sexo del acantocéfalo. Estos datos también se emplearon en un análisis de conglomerados, que mediante el índice de disimilitud de Distancia Euclidiana reflejó las relaciones de similitud morfológica entre las especies en un dendrograma construido por el método de neighbor joining (Sneath \& Sokal, 1973; Calle et al., 2008; Klingenberg et al., 2010).

Morfometría geométrica. Se analizó la probóscide mediante análisis de morfometría geométrica basada en puntos geométricos, y a partir de diferentes módulos del programa Integrated Morphometrics Package IMP6 y de la serie TPS (Zelditch et al., 2004), el cual genera un par de coordenadas (x, y) para cada marca registrada (Moreno-Barajas et al., 2013).

La forma de la probóscide se obtuvo mediante 2 landmarks en la base de la misma y 22 semilandmarks por todo el contorno de esta estructura, ubicados mediante 1 peine trazado con 2 puntos de apoyo localizados en la parte anterior y posterior (Fig. 2). Para todos los ejemplares analizados, se utilizaron los programas Makefan6 y tpsDig2.

Para evaluar la variación en la forma de la probóscide, las configuraciones de las marcas de cada organismo se ajustaron mediante el análisis de Procrustes en el módulo CoordGen6f, con la finalidad de eliminar toda la información no relacionada con la forma; posteriormente, se alinearon con el método de superimposición de Procrustes y un protocolo de alineación con el programa Semiland6, para eliminar el efecto de la ubicación, orientación y el tamaño (Moreno-Barajas et al., 2013). Los datos obtenidos se trabajaron con un Análisis de Componentes Principales en el software PCAGen6p, para identificar los caracteres que aportan mayor variación intraspecífica; además, permitió visualizar las variaciones de la forma mediante vectores y gradillas de deformación de cada grupo con la función de interpolación thin plate spline (Bookstein, 1991). 
Finalmente, con el software CVAGen6 se realizó un Análisis de Variables Canónicas con reducción a 2 CP, para identificar la posible segregación entre grupos en función del sexo del acantocéfalo, hospedero y microhábitat (Moreno-Barajas et al., 2013).

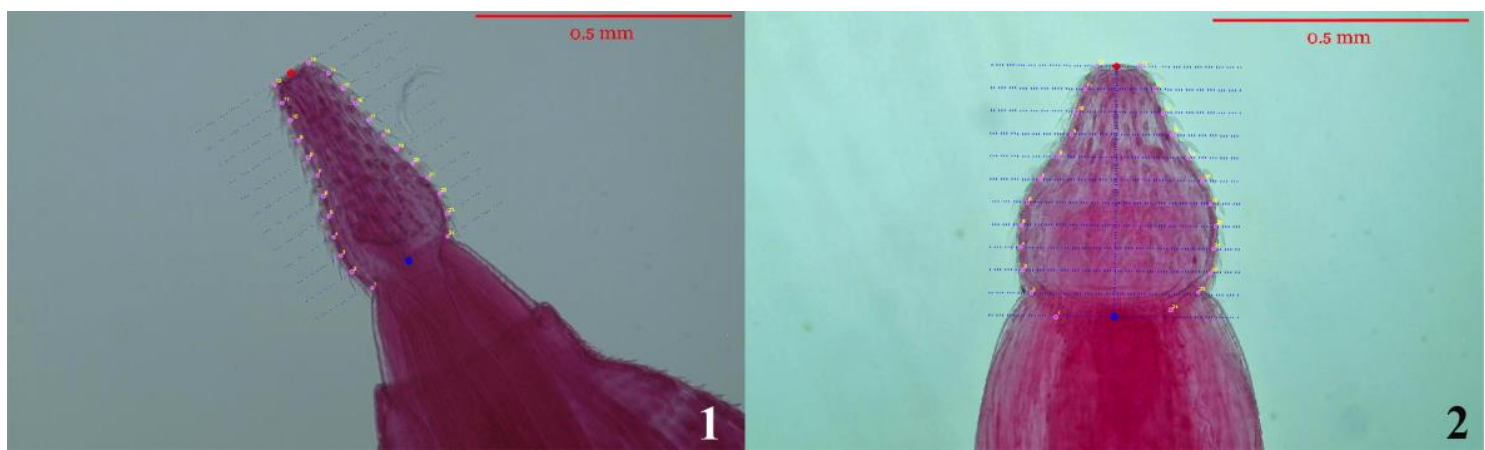

Figura 2. Colocación de los puntos geométricos en la probóscide: 2 landmarks y 22 semilandmarks alrededor de la probóscide de $P$. trochus machos (1) y hembras (2). Los semilandmarks se ubicaron a partir de 2 puntos de apoyo y 1 peine.

\section{RESULTADOS}

\section{Morfometría lineal}

De acuerdo con la dispersión de los datos mostrada en el Análisis de Componentes Principales (Fig. 3), los dos primeros componentes explican el $48.9 \%$ de la variación de los datos; gráficamente se observa una separación de acuerdo con el sexo de los organismos, en los que de acuerdo con el CP1 (26.4\%), las hembras presentan mayor longitud en los ganchos 9 y 10, así como en la base de los ganchos 8 y 10, mientras que los machos presentan menor longitud en el gancho 1 así como su base. El CP2 (22.5\%) indica que las hembras tienen una mayor anchura en la probóscide y los machos menor longitud en los ganchos 5, 6 y la base del gancho 1; el análisis no señala variación de acuerdo con el microhábitat. Posteriormente, el análisis de ANOVA (Fig. 4) demostró la existencia de diferencias significativas intersexos en el tamaño de los ganchos, siendo las hembras las que presentaron los de mayor tamaño. Entre los machos, se observa que los ubicados en el íleon mostraron los ganchos más grandes $\left(\mathrm{F}_{(5,28)} 6.52, p=0.0004\right)$.

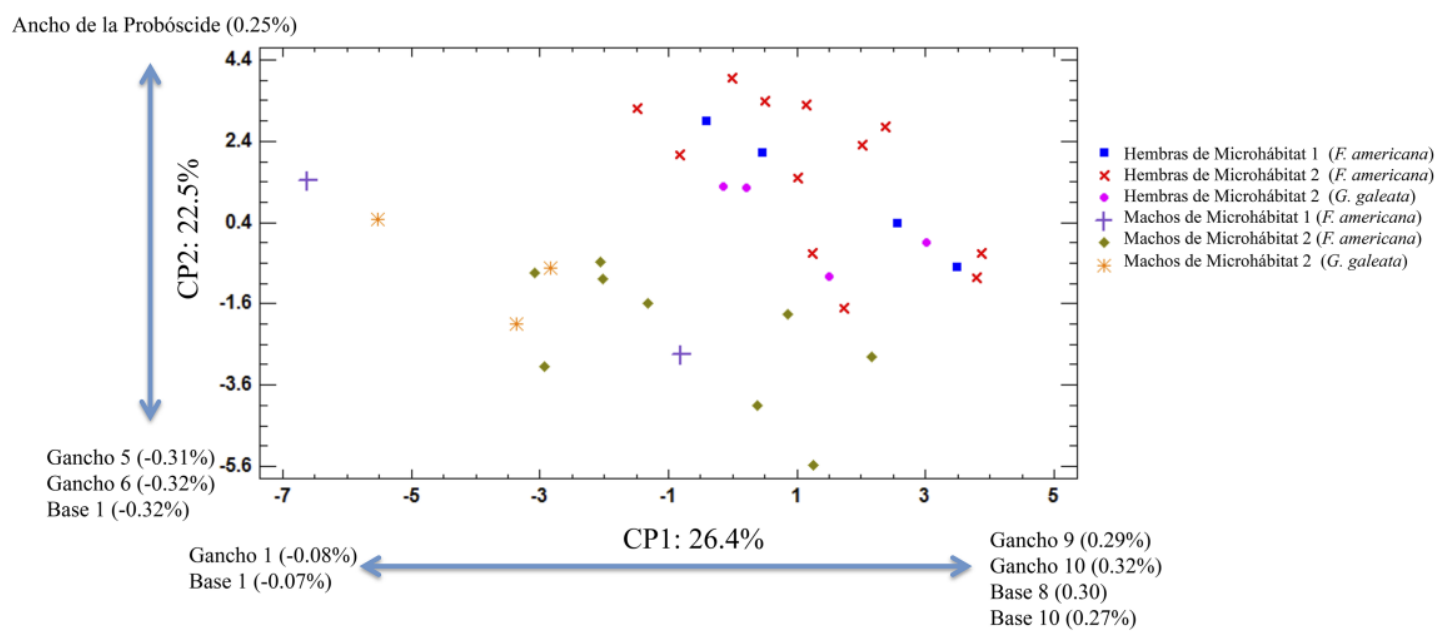

Figura 3. Variación de los datos de acuerdo con el Análisis de Componentes Principales. El CP1 explica 26.4\% y el $\mathrm{CP} 2$ el 22.5\%. Se notan agrupaciones de acuerdo con el sexo, en donde las hembras tienen las mayores cargas en las variables medidas. 
Caballero-Viñas et al.: Variación intraespecífica en la probóscide en Polymorphus trochus

En el dendrograma del análisis de conglomerados (Fig. 5), se observa la formación de cuatro grupos (Distancia Euclidiana $=0.75)$ con base en los ganchos y la probóscide; el grupo I (35.3\% del total) y II (29.4\%) está constituido por hembras, excepto los individuos 29 y 30 que son machos, los grupos III (29.4\%) y IV $(5.8 \%)$, se constituyen únicamente por machos. No se muestra una agrupación debido a la especie de hospedero ni al microhábitat

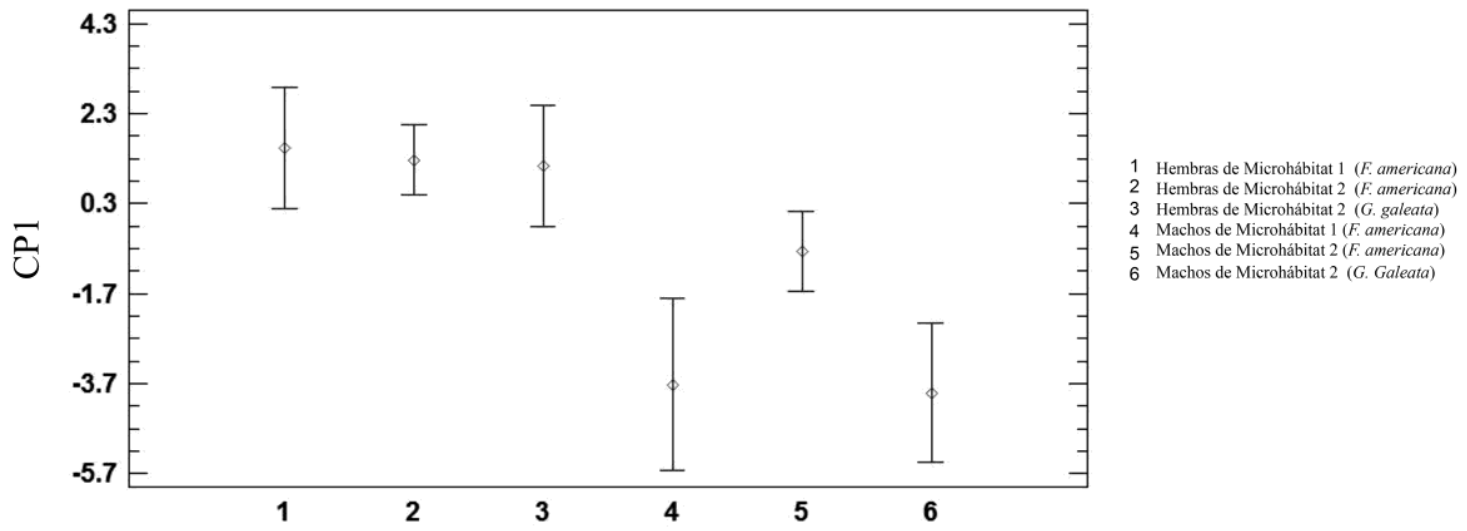

Figura 4. Comparación intragrupos de medias \pm DE del CP1, componente compuesto por la contribución parcial de cada variable morfométrica medida.

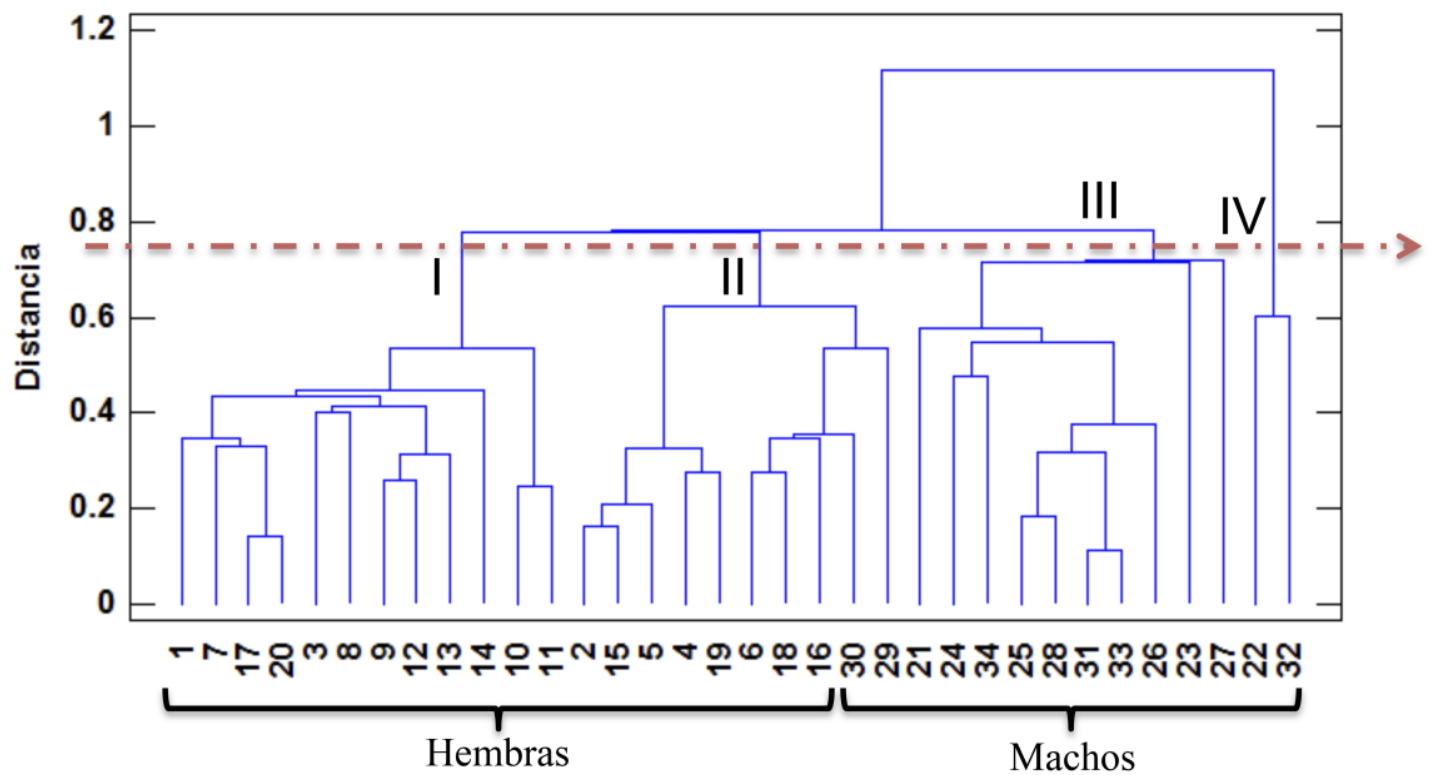

Figura 5. Dendrograma que organiza las variables morfológicas de los individuos medidos de acuerdo con el tamaño de los ganchos y la probóscide. La línea punteada marca la Distancia Euclidiana a la que se forman cuatro grupos (números romanos). 


\section{Morfometría geométrica}

Los morfotipos obtenidos a partir de las gradillas de deformación indican que el cambio en la forma de la probóscide está relacionado con el grosor de la base a la parte media de esta estructura. En las hembras del microhábitat 2 de ambas especies de hospederos, este ensanchamiento es más pronunciado y direccionado hacia el centro de esta estructura; el resto de los organismos (machos y hembras del microhábitat 1) muestran desplazamientos hacia el exterior de la probóscide. Con respecto al tamaño, los desplazamientos hacia el exterior son más pronunciados en los gusanos encontrados en el microhábitat 2 y más aún en aquellos de $G$. galeata, por lo que tienden a ser de mayor tamaño (Fig. 6). El Análisis de Componentes Principales indica que los dos primeros componentes explican más del $78 \%$ de la varianza de la forma de las probóscides analizadas (Fig. 7).
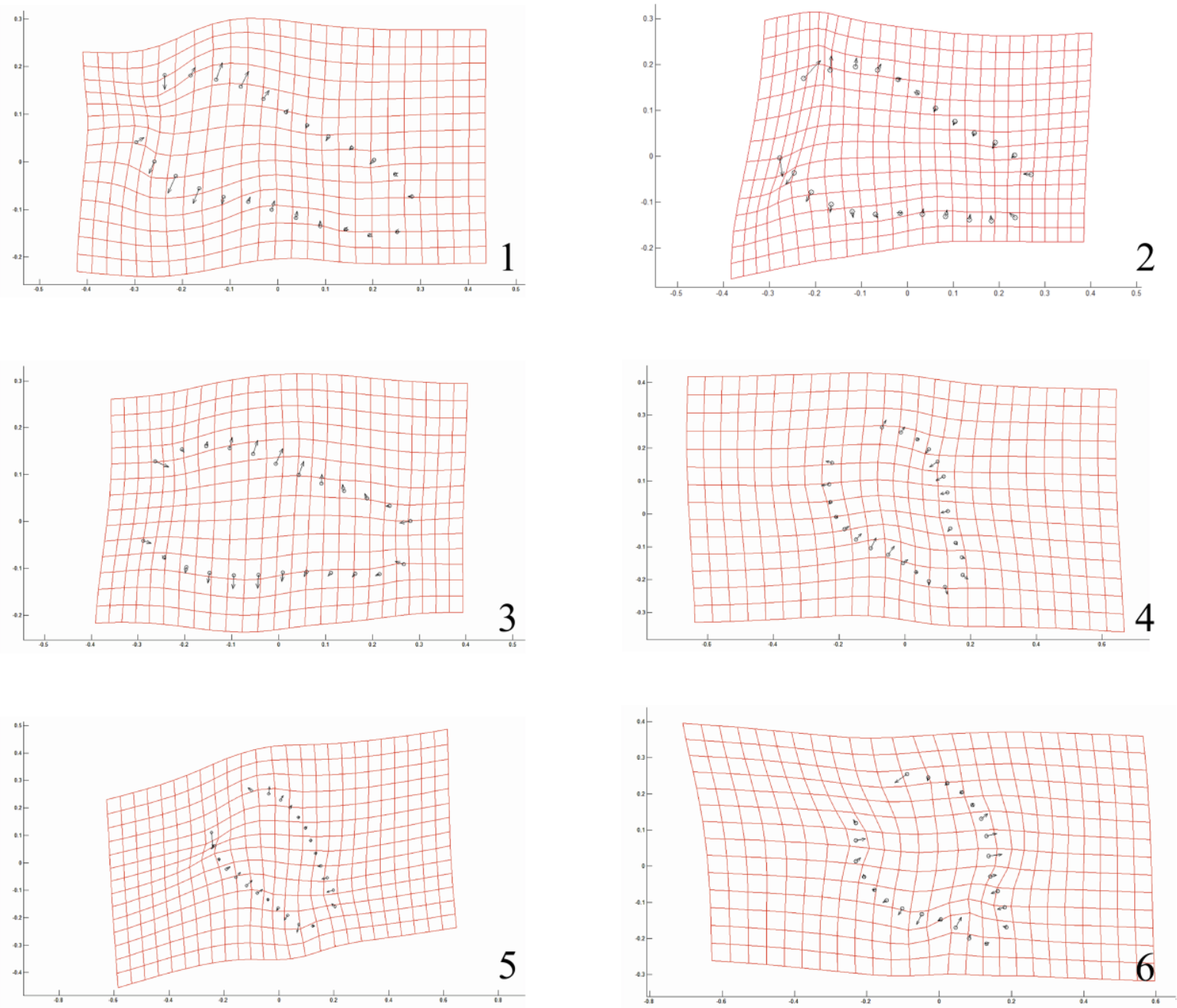

Figura 6. Gradillas de deformación de la probóscide de $P$. trochus por sexo, microhábitat y hospedero (1: machos de yeyuno de $F$. americana; 2 : hembras de yeyuno de $F$. americana, 3 : machos de íleon de $F$. americana; 4 : hembras de íleon de F. americana; 5: machos de íleon de G. galeata; 6: hembras de íleon de G. galeata). Los puntos indican la media, y las flechas la deformación de la estructura. 
Caballero-Viñas et al.: Variación intraespecífica en la probóscide en

Polymorphus trochus

El Análisis de Variables Canónicas (VC1: 5.1224 y VC2: 1.2157; eje1: $\lambda=0.0464, \chi^{2}=135.0723$, $\mathrm{df}=30, p=2.77556 \mathrm{e}-015$; eje $\left.2: \lambda=0.2843, \chi^{2}=55.3462, \mathrm{df}=20, p=3.64449 \mathrm{e}-005\right)$ separa los datos en dos grupos, de acuerdo con el microhábitat y el sexo. El Grupo 1 (a la derecha de la VC1) está integrado por hembras del microhábitat 1 de $F$. americana y todos los machos de ambos hospederos en ambos microhábitats. En el Grupo 2 (izquierda de la VC1), se ubican las hembras colectadas en el microhábitat 2 de ambos hospederos (Fig. 8).

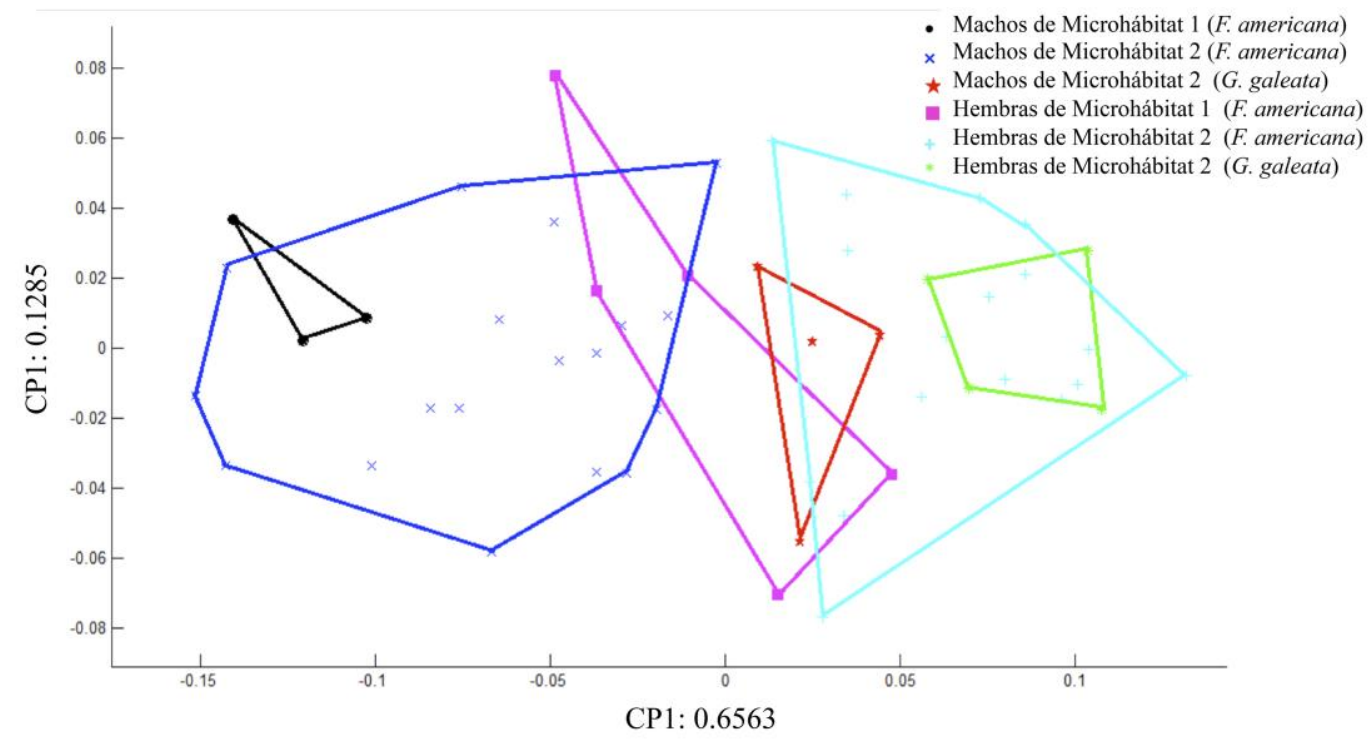

Figura 7. Análisis de Componentes Principales que indica la variación de la forma de la probóscide de $P$. trochus por sexo, microhábitat y hospedero.

\section{DISCUSIÓN}

El presente estudio sugiere que existen variaciones morfológicas de la probóscide de los acantocéfalos de acuerdo con el sexo, lo que confirma de manera analítica el dimorfismo característico de la especie registrado por Van Cleave (1945); sin embargo, este autor únicamente indica diferencias en la forma, mientras que en este estudio se observa que existe también dimorfismo de acuerdo con el tamaño de los ganchos. El microhábitat también modificó la forma de esta estructura, ya que el grupo de hembras encontradas en el íleon muestran morfotipos distintos a todos los machos y a las hembras encontradas en el yeyuno.

La similitud en la probóscide de machos con las hembras del yeyuno está moldeada por el desarrollo del gusano y los procesos reproductores, ya que diversas especies (como es el caso de los géneros Corynosoma, Moniliformis, entre otros) presentan patrones de migración a través del lumen intestinal hacia el yeyuno inferior y el íleon, mientras maduran, copulan y ovipositan; por lo tanto, las formas observadas, pueden ser el resultado de dinámicas de fijación, competencia intraespecífica o ambas, hasta llegar a su fijación permanente en el intestino (George-Nascimento \& Marin, 1992; Aznar et al., 2004). Esto se complementa con las fases reproductoras que se observaron para las hembras localizadas en el yeyuno, las cuáles presentaron estructuras reproductoras en diferentes estadios de maduración y de menor tamaño, mientras que las encontradas en el íleon tenían el aparato reproductor completamente desarrollado y en su mayoría se encontraban grávidas (Abele \& Gilchrist, 1977; Aznar et al., 2001). 
Por otra parte, se ha descrito en estudios previos que las hembras jóvenes al madurar, trasladarse y establecerse en el íleon, modifican la estructura de fijación para permanecer en el hospedero definitivo el mayor tiempo posible (Petrochenko, 1956). En el presente estudio, dicha modificación consiste en un ensanchamiento de la probóscide, que da como resultado una forma abultada e irregular, capaz de mantener una sujeción mayormente efectiva en las paredes intestinales, que les permita a las hembras incubar y expulsar los huevos en este microambiente, y así iniciar nuevamente el ciclo de vida.

Los resultados del presente estudio concuerdan con lo observado en la probóscide de otras especies de acantocéfalos (e. g. Filicolis, Polymorphus y Pomphorhynchus spp.), los cuales experimentan cambios progresivos al establecerse en el hospedero definitivo, donde una hinchazón de tamaño considerable se presenta justo en la base de la estructura, lo que permite una inserción total en la pared intestinal (Van Cleave, 1952, Bush et al., 2001).

Con respecto a los machos, que son más activos ya que se mueven para alcanzar y copular a tantas hembras como sea posible (Parshad \& Crompton, 1981), presentan estructuras adaptadas a una activa fijación y desprendimiento de la pared intestinal para ir en contra del movimiento peristáltico y forma sigmoidea del intestino (Abele \& Gilchrist, 1977). Estos resultados permiten sugerir que tanto los machos como las fases inmaduras de las hembras, presentan morfologías de la probóscide similares como una adaptación a la migración dentro del intestino, hasta su fijación permanente.

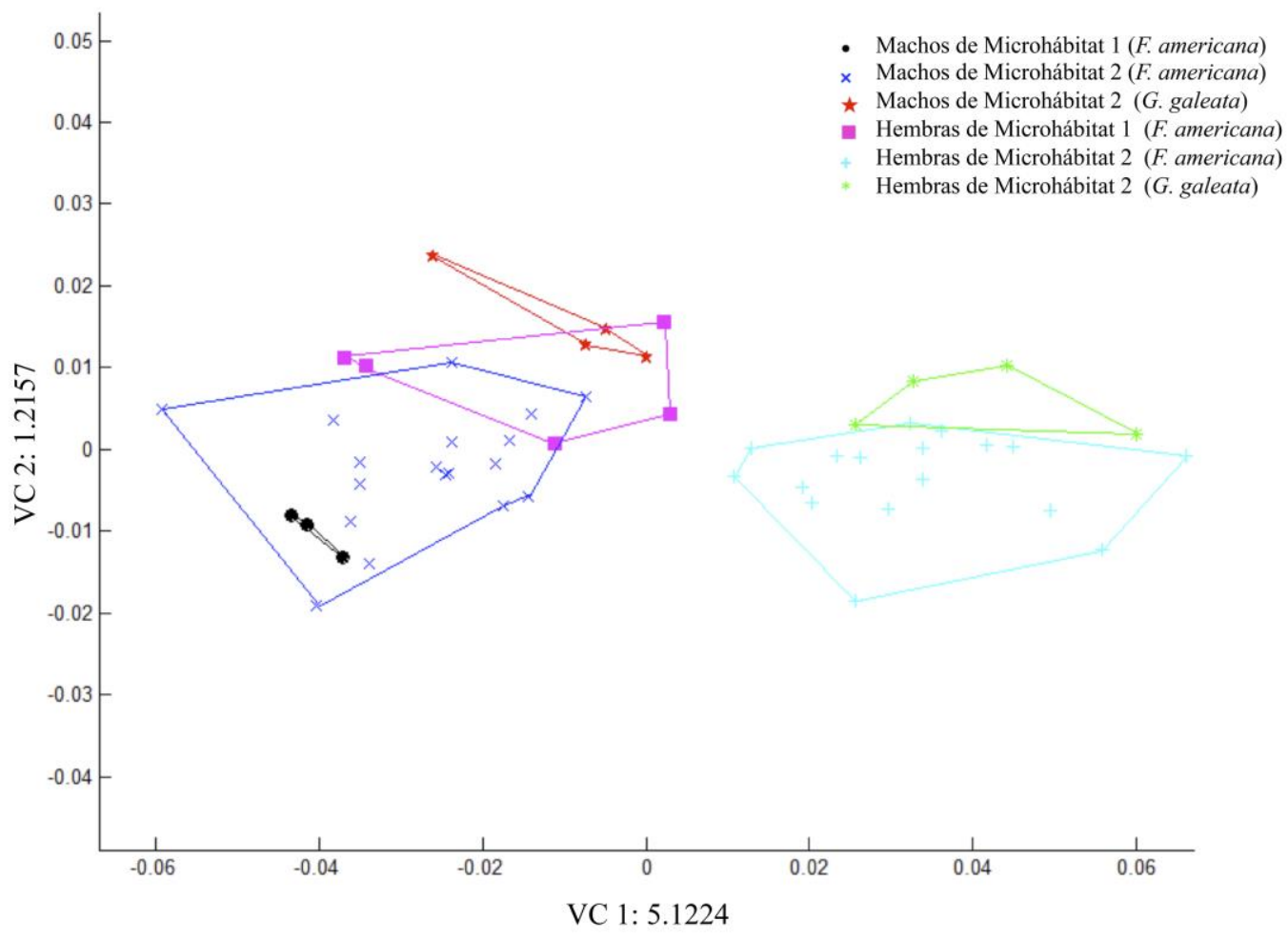

Figura 8. Análisis de Variables Canónicas con reducción a 2 Componentes Principales. Se agrupan los organismos de acuerdo con la forma, segregándose de acuerdo con el sexo y el microhábitat.

Por otro lado, los análisis de morfometría lineal no arrojaron variaciones en la longitud de los ganchos a nivel interhospedero, lo cual puede deberse a que, de acuerdo con lo reportado para varias especies, la inversión en la fijación se hace antes de establecerse en el hospedero definitivo, por lo tanto los 
Caballero-Viñas et al.: Variación intraespecífica en la probóscide en

Polymorphus trochus

ganchos están casi o completamente desarrollados en la fase de cistacanto dentro del hospedero intermediario, quizás como una prioridad de inversión energética del gusano en el desarrollo para asegurar el establecimiento exitoso al llegar al hospedero definitivo (Van Cleave, 1952; Petrochenko, 1956; Podesta \& Holmes, 1970; Miller \& Dunagan, 1985; Taraschewski, 2000) lo cual está en proceso de validación para esta especie.

La variación intraespecífica sugiere que $P$. trochus desarrolla estructuras de fijación de acuerdo con los requerimientos de fijación permanente del parásito dentro de los diferentes microhábitats. Además, los análisis geométricos fueron más significativos que los lineales al separar las hembras y los machos con respecto al microambiente y fase reproductora. Finalmente, en este estudio se comprueba de manera analítica que el dimorfismo sexual en esta especie no se limita a la forma, como tradicionalmente se ha descrito, sino que también debe considerarse el tamaño y la forma de la probóscide, así como la longitud de los ganchos.

AgRAdecimientos. Al Dr. Guillermo Salgado Maldonado, por su colaboración en la parte de técnicas helmintológicas e identificación de las especies de acantocéfalos, al Consejo Nacional de Ciencia y Tecnología por el otorgamiento de la beca nacional No. 252123, y a la Universidad Autónoma del Estado de México por el financiamiento 3831/2014/CIA otorgado para este estudio.

\section{LITERATURA CITADA}

Abele, L. G., Gilchrist, S. (1977) Homosexual Rape and sexual selection in Acanthocephalan worms. Science, 197 (4298), 81-3.

Amin, O. M. (1992) Review of the genus Polymorphus Luhe, 1911 (Acanthocephala:Polymorphidae), with the synonymization of Hexaglandula Petrochenko, 1950, and Subcoryno soma Hoklova, 1967, and a key to the species. Qatar University Science Journal, 12, 115-123.

Aznar, F. J., Bush, A. O., Fernandez, M., Raga, J. A. (1999) Constructional morphology and mode of attachment of the trunk of Corynosoma cetaceum (Acanthocephala: Polymorphidae). Journal of Morphology, 241, 237-249.

Aznar, F. J., Bush, A. O., Balbuena, J. B., Raga, J. A. (2001) Corynosoma cetaceum in the stomach of franciscanas, Pontoporia blainvillei (Cetacea): an exceptional case of habitat selection by an acanthocephalan. Journal of Parasitology, 87 (3), 536-541. https://doi.org/10.1645/0022-3395(2001)087[0536:CCITSO]2.0.CO;2

Aznar, F. J., Bush, A. O., Raga, J. A. (2002) Reduction and variability of trunk spines in the acanthocephalan Corynosoma cetaceum: the role of physical constraints on attachment. Invertebrate Biology, 121 (2), 104-114.

Aznar, F. J., Cappozzo, H. L., Taddeo, D., Montero, F. E., Raga, J. A. (2004) Recruitment, population structure, and habitat selection of Corynosoma australe (Acanthocephala) in South American fur seals, Arctocephalus australis, from Uruguay. Canadian Journal of Zoology, 82, 726-733. https://doi.org/10.1139/z04-044

Bookstein, F. L. (1991) Morphometrics Tools for Landmarks Data: Geometry and Biology. Cambridge University Press, Cambridge.

Bush, S. E. (2009 Does behavioural flexibility facilitate host switching by parasites? Functional Ecology, 23, 578-586.

Bush, A. O., Fernández, J. C., Esch, G. W., Seed, J. R. (2001) Parasitism: the diversity and ecology of animal parasites. Cambridge University Press, United Kingdom.

Calle, D. A., Quiñones, M. L., Erazo, H. F., Jaramillo, N. (2008) Discriminación por morfometría geométrica de once especies de Anopheles (Nyssorhynchus) presentes en Colombia. Biomédica, 28, 371-385. 
Colón-Quezada, D. (2009) Composición de la dieta de otoño del pato mexicano (Anas diazi) en el vaso sur de las Ciénegas del Lerma, estado de México. Revista Mexicana de Biodiversidad, 80, 193-202.

Corti, M., Aguilera, M., Capanna, E. (2001) Size and size changes in the skull accompanying speciation of South American spiny rats (Rodentia: Proechimys spp.). Journal of Zoology, 253, 537-547.

George-Nascimento, M., Marin, S. (1992) Efecto de dos especies hospedadoras, el lobo fino austral Arctocephalus australis (Zimmerman) y el lobo marino común Otaria byronia (Blainvillei) (Carnivora; Otariidae), sobre la morfología y la fecundidad de Corynosoma sp. (Acanthocephala; Polymorphidae) en Uruguay. Revista Chilena de Historia Natural, 65, 183-193.

Hernández-Orts, J. S., Timi, J. T., Raga, J. A., García-Varela, M., Crespo, E. A., Aznar, F. J. (2012) Patterns of trunk spine growth in two congeneric species of acanthocephalan: investment in attachment may differ between sexes and species. Parasitology, 139, 945-955. https://doi.org/10.1017/S0031182012000078

Klingenberg, C. P., Gidaszewski, N. A. (2010) Testing and quantifying phylogenetic signals and homoplasy in morphometric data. Systematic Biology, 59, 245-261.

Martínez-Haro, M., Sánchez-Nava, P., Salgado-Maldonado, G., Rodríguez-Romero, F. J. (2012) Helmintos gastrointestinales en aves acuáticas de la subcuenca alta del río Lerma, México. Revista Mexicana de Biodiversidad, 83, 36-41.

McDonald, M. E. (1988) Key to Acanthocephala Reported in Waterfowl. US. Department of the Interior, Fish and Wildlife Service. Resource Publication 173, Washington, D. C.

Miller, D. M., Dunagan, T. T. (1985) Functional morphology, pp. 73-123. En: Crompton, D.W.T., Nickol, B.B. (Eds.). Biology of the Acanthocephala. Cambridge University Press, Cambridge.

Moreno-Barajas, R., Rodríguez-Romero, F., Velázquez-Rodríguez, A., Aragón-Martínez, A. (2013) Variación geográfica en Phrynosoma orbiculare (Sauria: Phrynosomatidae): análisis de las subespecies. Acta Zoológica Mexicana (nueva serie), 29 (1), 129-143.

Parshad, V. R., Crompton, D. W. T. (1981) Aspects of acanthocephalan reproduction. Advances in Parasitology, 19, 73-138. https://doi.org/10.1016/S0065-308X(08)60266-3

Petrochenko, V. I. (1956) Acanthocephala of domestic and wild animals (Vol. 1). Izdatel'stvo Akademii Nauk SSSR, Moscow. 435 pp.

Petrochenko, V. I. (1958) Acanthocephala of domestic and wild animals (Vol. 2). lzdat. Akad. Nauk SSSR, Moscow. 478 pp.

Podesta, R. B., Holmes, J. C. (1970) The life cycles of three polymorphids (Acanthocephala) occurring as juveniles in Hyalella azteca (Amphipoda) at Cooking Lake, Alberta. Journal of Parasitology, 56, $1118-1123$.

Poulin, R. (2007) Investing in attachment: evolution of anchoring structures in acanthocephalan parasites. Biological Journal of the Linnean Society, 90, 637-645. https://doi.org/10.1111/j.1095-8312.2006.00754.x

Preston, E. (1998) A field guide to the birds of Mexico and adjacent areas Belize, Guatemala and El Salvador. University of Texas, Austin. 209 pp.

Rohlf, F. J., Marcus, L. F. (1993) A revolution in morphometrics. Trends in Ecology \& Evolution, 8, 129132.

Salgado-Maldonado, G. (2009) Manual de prácticas de parasitología con énfasis en helmintos parásitos de peces de agua dulce y otros animales silvestres de México. Instituto de Biología, UNAM, Ciudad de México.

Sisson, S., Grossman, J. D. (2002) Anatomía de los animales domésticos (Tomo II). Elsevier. Barcelona, Madrid. 1046 pp.

Sneath, P. H. A., Sokal. R. R. (1973) Numerical taxonomy. The principles and practice of numerical classification. W. H. Freeman and Company, San Francisco. 573 pp.

Taraschewski, H. (2000) Host-parasite interactions in Acanthocephala: a morphological approach. Advances in Parasitology, 46, 1-179. 
Caballero-Viñas et al.: Variación intraespecífica en la probóscide en

Polymorphus trochus

Toro, I. M. V., Manriquez, S. G., Suazo, G. I. (2010) Morfometría geométrica y el estudio de las formas biológicas: de la morfología descriptiva a la morfología cuantitativa. International Journal of Morphology, 28 (4), 977-990.

Van Cleave, H. J. (1945) A new species of the Acanthocephalan genus Polymorphus from the American Coot. The Journal of Parasitology, 31 (2), 128-130.

Van Cleave, H. J. (1952) Some host-parasite relationship of the acanthocephala with special reference to the organs of attachement. Experimental Parasitology, 1 (13), 305-330.

Van Perlo, B. (2006) Birds of Mexico and Central America. Princeton University Press, New Jersey.

Yamaguti, S. (1963) Systema Helminthum. Acanthocephala (Vol. 5). New York, Interscience Publishers. $423 \mathrm{pp}$.

Zelditch, M., Donald, L., Davis, S., William, L. (2004) Geometric Morphometrics for Biologists: a primer. Elsevier Academic Press, USA. 443 pp. 